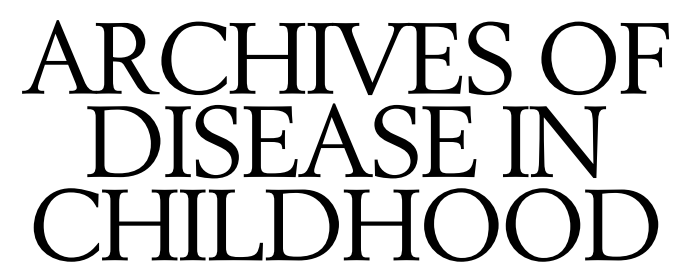

The Fournal of the Royal College of Paediatrics and Child Health

\title{
Annotations
}

\section{Genes and cancer}

In developed countries, approximately 1 in 600 children develop cancer before they are 15 years old. Half of all childhood malignancies are diagnosed during the first 5 years of life. About 33\% of cases are leukaemias, $25 \%$ brain tumours, and $10-12 \%$ lymphomas. The remaining cases are mainly embryonal tumours including neuroblastoma, Wilms's tumour, embryonal rhabdomyosarcoma, retinoblastoma, and hepatoblastoma. ${ }^{12}$ Many of the leukaemias and brain tumours also constitute embryonal neoplasms. The histological appearance of embryonal neoplasms resembles that seen in the developing embryo and fetus. The early onset of these neoplasms and their embryonal appearance strongly suggest that prenatal, including genetic, factors are important. This article considers the role of genetic predisposition in the development of childhood cancer.

Genetic predisposition to cancer may be considered under four areas:

- highly penetrant genes that give rise to distinct familial clusters of cancers with a clear pattern of inheritance

- genes that confer a lower penetrance, with most gene carriers remaining unaffected. Multiple case familial clusters would not arise but occasional cancer affected sibling pairs or parent-child pairs may occur. Such genes may well exist and could play an important role in causing childhood cancers. At present, however, none has been identified and consequently, their possible importance cannot be estimated

- various syndromes that confer an increased risk of childhood cancers but where congenital abnormalities represent the main manifestation of the genetic disorder

- normal polymorphic variants of genes may exist that confer an increased risk of developing childhood cancers by modifying response to environmental factors.

\section{High penetrance genes and familial cancer syndromes \\ These syndromes are characterised by a high incidence of specific cancers within an affected family. Usually, there are no associated non-neoplastic phenotypic markers of gene carrier status. There are several such syndromes which confer a high risk of specific paediatric cancers, and two examples are described below.}

RETINOBLASTOMA AND THE RB1 GENE

Retinoblastoma affects approximately 1 in 20000 liveborn children, and occurs in hereditary and sporadic forms. ${ }^{3}$ In contrast with other embryonal tumours, prognosis for retinoblastoma has been favourable since the practice of enucleation became common towards the end of the 19th century. Affected individuals therefore survived to reproductive age and it was recognised that the tendency to develop retinoblastoma could be inherited. Hereditary retinoblastoma is usually bilateral and often diagnosed during the first year of life. Multiple tumours within a single eye are common. Non-hereditary cases are invariably unilateral and unifocal with a somewhat later onset. In 1971, Knudson proposed that sporadic unilateral disease and heritable bilateral disease both arise after two mutational events occurring in a target cell. ${ }^{4}$ In sporadic non-heritable retinoblastoma both mutations occur somatically. In heritable cases the initial mutation occurs in the germline. Every cell therefore carries the mutation, giving a high probability of a second mutation in one or more target cells. Follow up studies of survivors show that patients with heritable disease are at increased risk of developing further malignancies, particularly osteosarcoma, soft tissue sarcoma, and melanoma. ${ }^{56}$

Studies of retinoblastoma have been extremely important in the development of ideas about genetic predisposition to cancer, including the recognition that a single gene can predispose to more than one type of cancer. The concept of tumour suppressor genes, whereby it is necessary for both copies of the normal wild-type allele to be lost by mutation or deletion for tumour development to proceed, grew out of the two mutation model. Thus, although a dominant pattern of inheritance is apparent at the clinical level, at the cellular level such genes are recessive.

LI-FRAUMENI SYNDROME AND THE TP53 GENE

The $\mathrm{Rb} 1$ gene was the first tumour suppressor gene to be cloned and the second was the TP53 gene. Germline mutations to TP53 give rise to familial clusters of cancers consistent with Li-Fraumeni syndrome (LFS). LFS is characterised by bone and soft tissue sarcoma, breast cancer, brain tumours, leukaemia, and adrenocortical carcinoma, with onset during childhood or early adult life. ${ }^{7}$ Recent studies show that at least $70 \%$ of families with clinical features of LFS carry germline TP53 mutations. ${ }^{8}$ The pattern of cancers and penetrance vary according to type of mutation. ${ }^{9}$

Historically, childhood onset tumours associated with LFS conferred a poor prognosis. Most gene carriers therefore would not have survived to reproductive age. It is thus difficult on the basis of epidemiological data to estimate the frequency of germline TP53 mutations among populations of children with these cancers. Mutation screening studies of mainly small series of patients indicate a frequency of 
5-50\% depending on tumour type, but it should be recognised that the techniques used in these studies would have missed some mutations. ${ }^{7}$ There is therefore a need for studies of large patient series using more extensive mutation analysis methods to determine the frequency of TP53 mutations among childhood tumours. Recognition of individuals with TP53 mutations could be important clinically because the presence of such a mutation may influence response to cytotoxic treatment. Furthermore, as with $\mathrm{Rb} 1$ mutations, the risk of developing second and subsequent malignancies is greatly increased in patients with TP53 mutations. ${ }^{9}$

\section{Congenital malformation syndromes associated with childhood cancers}

Childhood cancers, particularly embryonal tumours, might be regarded as developmental anomalies. Certain malformations and childhood cancers could arise therefore as a result of the same aberrant developmental processes. Several examples exist of malformation syndromes where this appears to be the case. In these syndromes, the tumour represents one of several associated features. Patients may display some, but not all, of the syndrome characteristics.

WILMS'S TUMOUR, WAGR SYNDROME, DENYS-DRASH

SYNDROME, AND THE WT1 GENE

Wilms's tumour is an embryonal tumour arising in the developing kidney which affects approximately 1 in 10000 liveborn children. Children with Wilms's tumour experience an excess of various congenital anomalies including bilateral, congenital aniridia. ${ }^{10}$ Children with aniridia often also display genitourinary abnormalities and mental retardation. The association of Wilms's tumour with these abnormalities is known as WAGR syndrome. In the majority of patients with WAGR syndrome, a constitutional heterozygous deletion involving the short arm of chromosome $11[11 \mathrm{p} 13]$ can be detected. ${ }^{11}$ In patients with such deletions, aniridia is fully penetrant and Wilms's tumour develops in up to $50 \%$ of cases. A gene designated WT1, which is important in kidney development, was isolated from this chromosome region. Whereas deletions involving WT1 give rise to WAGR syndrome, specific constitutional point mutations within WT1 give rise to Denys-Drash syndrome (DDS). DDS is characterised by severe nephropathy, which can be fatal, dysgenetic male pseudohermaphroditism, and Wilms's tumour. Thus WT1 is involved in both syndromes, but the phenotype depends on the nature of the mutational event. ${ }^{12}$

\section{CONGENITAL OVERGROWTH SYNDROMES AND EMBRYONAL \\ TUMOURS}

Several overgrowth syndromes are associated with increased risk of embryonal tumours. The best known is Beckwith-Weidemann syndrome (BWS). The principal characteristics of BWS are prenatal and postnatal gigantism, macroglossia, abdominal wall defects, visceromegaly, muscular hypertrophy, advanced bone age, craniofacial and ear anomalies, and neonatal hypoglycaemia. The overgrowth may affect only part of the body (hemihypertrophy). Usually, children with BWS are developmentally normal, ${ }^{13}$ and about $8 \%$ develop tumours. The most common malignancy is Wilms's tumour, but hepatoblastoma and adrenocortical carcinoma are also relatively frequent. Other neoplasms seen in association with BWS include rhabdomyosarcoma, neuroblastoma, and pancreaticoblastoma.

The phenotype can be variable, with some children only mildly affected. Risk of malignancy, however, appears to be similar in children with incomplete BWS, and those showing all principal features but presence of hemihypertrophy may confer a higher risk. ${ }^{14}{ }^{15}$ Most cases of BWS are sporadic, but familial cases showing autosomal dominant inheritance occur. Linkage studies indicate that the locus for BWS is within chromosome $11 \mathrm{p} 15$. Although a number of candidate genes have been proposed, the BWS gene has not yet been characterised. ${ }^{16}$

More recently, other overgrowth syndromes which appear to predispose to embryonal tumours have been recognised, including Simpson-Golabi-Behmel syndrome (SGBS) and Perlman's syndrome. SGBS shares many features with BWS, but in children with SGBS cleft lip and palate, cardiac and skeletal anomalies, and developmental delay are common features. SGBS is X-linked and the gene responsible, which maps to chromosome Xq26, is a glypican gene designated GPC $3 .^{17}{ }^{18}$ The frequency of SGBS among children with embryonal tumours and the risk of neoplasia among children with SGBS has yet to be clarified. In Perlman's syndrome there is a high associated neonatal mortality rate and mental retardation. Renal abnormalities are observed and there is an apparently high incidence of Wilms's tumour, often bilateral, during the first year of life. Perlman's syndrome shows autosomal recessive inheritance. ${ }^{19}$

Increased action of insulin-like growth factor 2 (IGF2) has been implicated in the overgrowth which characterises BWS, and it has been suggested that the GPC3 gene might also interact with IGF2. Common developmental processes may therefore exist, leading to these overgrowth syndromes and associated embryonal tumours. ${ }^{17}{ }^{18}$

\section{Interaction of genetic and environmental factors in childhood cancer aetiology}

Various ways exist in which individual susceptibility to carcinogenic effects of environmental agents may depend on genetically determined factors. In adults, it is established that various drug metabolising enzymes play an important part in determining the carcinogenicity of certain chemicals. Normal polymorphic variation can lead to differences in the way or extent to which individuals metabolise chemical agents. An example of this, involving the cytochrome p450 system, is the ability to metabolise debrisoquine. Poor metabolisers appear to have a lower risk of lung and bladder cancers. ${ }^{20} 21$

TRANSPLACENTAL CARCINOGENESIS AND GENETIC VARIATION IN SUSCEPTIBILITY

Epidemiological studies have indicated an increased risk of certain childhood cancers in the offspring of individuals in occupations associated with exposure to potentially mutagenic chemicals during a child's prenatal life. Additionally, some studies appear to show an association between maternal diet during pregnancy and risk of cancer in offspring. ${ }^{22}$ Although there is variation and inconsistency in results between these studies, the overall implication is that transplacental carcinogens may have a role in the aetiology of some childhood cancers. If this is the case, then childhood cancer risk may be affected by genetically determined variation in ability to metabolise transplacental carcinogens in both mother and fetus. In experimental animals, it has been shown that cancer incidence in offspring transplacentally exposed to carcinogens depends on the genotype of the mother and the fetus with respect to genes involved in carcinogen metabolism. ${ }^{23}$ It is possible that this type of mechanism also operates in humans. Although this is speculative at present, it does suggest potential lines of research.

CHILDHOOD LEUKAEMIA, INFECTIONS, AND HLA GENOTYPE There is mounting epidemiological evidence to suggest that childhood leukaemia arises as a rare outcome of a common infection. ${ }^{24}$ If infections are involved in the 
aetiology of childhood leukaemia, then it might be predicted that risk will depend on an individual's HLA genotype because of the role of HLA in control of immune response. Such an association would provide an example of genetically determined susceptibility to an environmental risk factor. Recent work suggests that this may be the case. Taylor et al have demonstrated a higher frequency of specific HLA class II alleles in children with c-ALL compared with controls. ${ }^{25}$ Furthermore, their results indicate that the effect may be more marked in boys than in girls. This work provides an example of geneticenvironmental interaction in childhood malignant disease.

\section{Conclusions}

Genetic susceptibility is important in childhood cancer aetiology. More is known about genes conferring high risk than genes with lower penetrance. On the basis of present knowledge, the former may account for 5-10\% of childhood cancers. The true incidence of childhood cancers arising through mutations in such genes is not known with any accuracy, and this figure is likely to be an underestimate. Results of current studies may show that low penetrance genes are more important numerically. The understanding of genetic susceptibility to childhood cancer is important scientifically and clinically and may eventually lead to intervention and preventative measures.

CRC Paediatric and Familial Cancer Research Group,

JLLIAN M BIRCH

Royal Manchester Children's Hospital,

Stancliffe, Hospital Road, Manchester M27 4HA, UK

1 Blair V, Birch JM. Patterns and temporal trends in the incidence of malignant disease in children: I. Leukaemia and lymphoma. Eur 7 Cancer 1994;30A: $1490-8$

2 Blair V, Birch JM. Patterns and temporal trends in the incidence of malignant disease in children: II. Solid tumours of childhood. Eur $\mathcal{F}$ Cancer malignant disease in

3 Cowell John K. Retinoblastoma: the paradigm for a genetically inherited cancer syndrome In: Eeles RA, Ponder BAJ, Easton DF, Horwich A, eds. Genetic predisposition to cancer. London: Chapman \& Hall Medical, 1996.

Genetic predisposition to cancer. London: Chapman \& Hall Medical, 1996.
Knudson AG. Mutation and cancer: statistical study of retinoblastoma. Proc

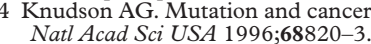

5 Draper GJ, Sanders BM, Kingston JE. Secondary primary neoplasms in patients with retinoblastoma. Br f Cancer 1996;53:661-71.
6 Schottenfeld D. Multiple primary cancers. In: Schottenfeld D, Fraumeni JF, eds. Cancer epidemiology and prevention. 2nd ed. Oxford: Oxford University Press, 1996.

7 Varley JM, Evans DGR, Birch JM. Li-Fraumeni syndrome: a molecular and clinical review. Br f Cancer 1997;76:1-14.

8 Varley JM, McGown G, Thorncroft M, et al. Germ-line mutations of TP53 in Li-Fraumeni families: an extended study of 39 families. Cancer Res 1997;57:3245-52.

9 Birch JM, Blair V, Kelsey AM, et al. Cancer phenotype correlates with constitutional TP53 genotype in families with the Li-Fraumeni syndrome. Oncogene 1998;17:1061-8.

10 Birch JM, Breslow N, Epidemiologic features of Wilms' tumour. Haematology/Oncology Clinics of North America 1995;9:1157-78.

11 Riccardi VM, Sujansky E, Smith AC, Francke U. Chromosomal imbalance in the aniridia-Wilms' tumor association: $11 \mathrm{p}$ interstitial deletion. Pediatrics 1978;61:604-10.

12 Huff V. Genotype/phenotype correlations in Wilms' tumor. Med Pediatr Oncol 1996;27:408-14.

13 Elliott M, Bayly R, Cole T, Temple IK, Maher ER. Clinical features and natural history of Beckwith-Wiedemann syndrome: presentation of 74 new cases. Clin Genet 1994; 46:168-74.

14 Pettenati MJ, Haines JL, Higgins RR, Wappner RS, Palmer CG, Weaver DD. Wiedemann-Beckwith syndrome: presentation of clinical and cytogenetic data on 22 new cases and review of the literature. Hum Genet 1986;74:143 54 .

15 Wiedemann HR. Tumours and hemihypertrophy associated with Wiedemann-Beckwith syndrome. Eur f Pediatr 1983;141:129.

16 O'Keefe D, Dao D, Zhao L, Sanderson R, Warburton D, Weiss L, et al. Coding mutations in p57KIP2 are present in some cases of BeckwithWiedemann syndrome but are rare or absent in Wilms' tumors. Am f Hum Genet 1997;61:295-303.

17 Pilia G, Hughes-Benzie RM, MacKenzie A, et al. Mutations in GPC3, a glypican gene, cause the Simpson-Golabi-Behmel overgrowth syndrome. Nat Genet 1996;12:241-7.

18 Hughes-Benzie RM, Pilia G, Xuan JY, et al. Simpson-Golab-Behmel syndrome: genotype/phenotype analysis of 18 affected males from 7 unrelated families. Am F Med Gen 1996;66:227-34

19 Grundy RG, Pritchard J, Baraitser M, Risdon A, Robards M. Perlman and Wiedemann-Beckwith syndromes: two distinct conditions associated with Wilms' tumour. Eur f Pediatr 1992;151:895-8.

20 Ayesh R, Idle JR, Ritchie JC, Crothers MJ, Hetzel MR. Metabolic oxidation phenotypes as markers for susceptibility to lung cancer. Nature 1984;312: 169-70.

21 Gough AC, Miles JS, Spurr NK, et al. Identification of the primary gene defect at the cytochrome P450 CYP2D locus. Nature 1990;347:773-6.

22 Chow WH, Linet MS, Liff JM, Greenberg RS. Cancers in children. In: Schottenfeld D, Fraumeni Jr JF. 2nd ed. Cancer epidemiology and prevention. Oxford: Oxford University Press, 1996.

23 Anderson LM, Jones AB, Miller MS, Chauhan DP. Metabolism of transplacental carcinogens. In: Napalkov NP, Rice JM, Tomatis L, Yamasaki H, eds. Metabolism of transplacental carcinogens in perinatal and multigeneration carcinogenesis. Lyon: IARC Scientific Publications, 1989:155-88.

24 Greaves MF. Aetiology of acute leukaemia. Lancet 1997;349:344-9.

25 Taylor GM, Dearden S, Payne N, et al. Evidence that an HLA-DQA1 and DQB1 haplotype influences susceptibility to childhood common ALL in males provides further support for an infection-related aetiology. $\mathrm{Br} \mathcal{F}$ Cancer 1998;75:561-5.

\section{Umbilical cord blood transplantation}

Since Gluckman et al's first description of successful haemopoietic stem cell transplantation using umbilical cord blood (UCB) as the source of marrow progenitors in $1989,{ }^{1}$ more than 500 umbilical cord blood stem cell transplants have been performed. Umbilical cord blood banks have been set up in the USA and Europe to store these cells, which in the past have been considered a waste product of reproduction. Although most UCB haemopoietic stem cell transplants have taken place in the past three years, recently published reports ${ }^{2-5}$ have given an important insight into the clinical potential of UCB as a source of haemopoietic progenitor cells for transplantation.

Allogeneic bone marrow transplantation (BMT) can potentially be used to cure a variety of diseases including haematological malignancies, bone marrow failure syndromes, haemoglobinopathies, immunodeficiencies, and some inborn errors of metabolism. ${ }^{6}$ The use of allogeneic BMT is limited by the need for adequate tissue matching of host and donor cells to reduce the risks of rejection and the severity of graft versus host disease (GVHD) in the short term, while allowing immune reconstitution in the longer term. Many patients who might benefit from allogeneic
BMT are prevented from doing so because there is no adequately matched donor available. In part, this problem has been addressed by the establishment of large panels of unrelated adult donors who are prepared to donate their bone marrow. Approximately five million donors are available worldwide. Despite these large numbers the need for precise tissue matching compounded by the predominantly white European ethnicity of the donor panels means that a significant number of patients remain unable to benefit from BMT. There is a need for a source of haematopoietic stem cells with a less rigorous requirement for tissue matching that could be used for transplantation into patients who do not have a conventional donor. UCB cells are possible candidates for this clinical role.

\section{Umbilical cord blood}

Laboratory studies ${ }^{7-9}$ have demonstrated and clinical use confirmed that UCB is a rich source of haemopoietic stem cells. UCB contains an increased proportion of the earliest progenitors, and per nucleated cell UCB has approximately 10 times the repopulation potential of bone marrow. Moreover, in vitro studies ${ }^{10-13}$ have suggested that naive 
UCB lymphocytes are potentially less immunologically active than those usually found in the blood or marrow and may therefore produce fewer problems with GVHD than functionally mature lymphocytes harvested from live donors.

\section{Harvesting umbilical cord cells}

Umbilical cord cells can be harvested following vaginal delivery by cannulation of the placental vessels with the placenta still in utero or by direct expression of cord blood from the placenta following its delivery (usually in a room adjacent to the delivery suit) or at the time of caesarean section. There appears to be little difference in the quality of cells harvested by either method following vaginal delivery but cells from caesarean section may be less satisfactory ${ }^{14}$; however, these recent data are not borne out by an earlier study. ${ }^{15}$ A significant minority of postplacental delivery harvests provide an inadequate volume of UCB and are discarded. In Europe it is considered best practice that where possible UCB is harvested by specially trained staff working for the cord blood banks. However, in the USA harvesting by different obstetric teams has not been associated with a reduction in UCB quality. ${ }^{16}$

For directed family donations, cannulation of the placental vessels in utero is the preferred option to reduce the risk of an inadequate harvest. Where UCB is being donated for unrelated use postplacental delivery harvesting is more appropriate as it is less intrusive and reduces the need for donor counselling. Only mothers who produce adequate UCB harvests need to be approached for permission to store and test the UCB and to have a medical history taken. This history is relied on to ensure that the cord blood is unlikely to carry any known genetic disorders. The chances of a fetus carrying an unknown but clinically significant congenital disorder is negligible.

A very important part of the procedure is to ensure that the midwife or obstetrician attending the delivery does not clamp the cord earlier than usual just because the UCB is to be harvested. This prevents any potential harm to the fetus. Harvests over $40 \mathrm{ml}$ are considered suitable for use. Usually between 40 and $150 \mathrm{ml}$ are obtained, which approximates to between 4 and $11 \times 10^{8}$ nucleated cells available for transplantation.

Samples are taken at the time of harvesting from the mother for microbiological screening, including HIV I and II, hepatitis B and C, and syphilis. Retesting of the donor for HIV at a later date is not performed because the risk of silent HIV infection at the time of delivery is extremely small, and failure to locate donors would lead to an unacceptable waste of stored harvested cord bloods. ${ }^{17}$ As well as being tissue typed, a specimen of cord blood is often kept aside for HIV retesting should the UCB be required for transplantation. Further tests at the time of transplantation are probably unnecessary and are not performed on other types of stem cells stored in liquid nitrogen before use in transplantation. The UCB may be volume reduced but is most often directly mixed with DMSO to allow for long term storage in liquid nitrogen following a brief period in a quarantine freezer awaiting the results of virology tests. The UCB is also tested for bacteria. Improved harvest techniques have reduced the incidence of bacterial contamination. ${ }^{18}$

The costs involved in harvesting and banking UCB are not insignificant. If it is to be done properly unrelated cord blood banking requires a considerable outlay in equipment, storage facilities, and trained staff. Each cord harvest costs approximately $£ 2500$.

\section{Theoretical advantages and disadvantages of UCB} transplantation

The advantages to the donor are obvious. As long as care is taken to clamp the cord at the normal time the fetus will come to no harm. There is no need to anaesthetise the donor. The donor feels no discomfort and does not require time off work or school.

Potential advantage for the recipient is crucially dependent on the theoretical immune tolerance of UCB lymphocytes that might allow for transplantation across presently insurmountable HLA barriers. If successful engraftment is possible across HLA barriers and GVHD is controllable, the potential donor pool will be greatly increased and many more patients will be able to undergo curative haemopoietic stem cell transplantation. There are other possible advantages. UCB cells are potentially immediately available. Thus the time from consideration of transplantation to the actual procedure should be reduced compared to donation from an unrelated panel donor. This may be important for patients with malignancies and some inborn errors of metabolism where delays in transplantation may adversely affect outcome. The risk of transmissible infection should also be less. The UCB comes already virologically tested and will be free from postnatally acquired infections such as cytomegalovirus.

There are disadvantages. The reduced number of stem cells present in the UCB may limit their use to small recipients. The theoretical immune naivete of the transplanted lymphocytes may reduce the graft versus leukaemia effect of the UCB transplants with an increased risk of relapse in patients transplanted for malignancy. Where relapse does occur the donor is not available to donate peripheral blood lymphocytes that could be used to treat the relapse.

\section{Clinical results}

\section{DIRECTED (FAMILY) DONATION}

Eurocord ${ }^{4}$ has recently reported the results of 78 related UCB transplants performed in Europe between October 1988 and December 1996 with a median follow up of 29 months. The patients were mainly children; median age was 5 years (range 0.2 to 20) and median weight was $19 \mathrm{~kg}$ (range 5 to 50). Thirty eight patients had leukaemia. In 60 cases the donor was an HLA identical sibling and in 18 there was a degree of HLA disparity. Induction regimens were standard with 36 patients receiving cyclophosphamide and total body irradiation, and 40 chemotherapy alone, which included busulphan. Post-transplant immunosuppression was with cyclosporin alone or in combination with prednisolone or methotrexate or both. Only four patients received serotherapy with either antithymocyte globulin or monoclonal antibodies.

Overall 53 patients survived one year; 44 in the HLA matched group and six in the HLA mismatched group. In 10 patients receiving UCB mismatched at three or more HLA antigens only one survived for 12 months. Neutrophil engraftment was better in patients younger than 6 years of age and weighing less than $20 \mathrm{~kg}$. Thirty five of 48 patients receiving $<3.7 \times 10^{7}$ cells $/ \mathrm{kg}$ had neutrophil engraftment compared with 34 of 40 who received $>3.7 \times 10^{7}$ cells $/ \mathrm{kg}$. Platelet engraftment was also favoured by age under 6 years and weight more than $20 \mathrm{~kg}$. HLA disparity was significantly associated with poor platelet engraftment. Acute GVHD developed to grade II or more in $9 \%$ of the fully matched recipients and in nine of the mismatched transplants. $14.3 \%$ of evaluable patients went on to develop chronic GVHD. Ten of the 38 patients with leukaemia relapsed during the median follow up of 29 months. Survival was also significantly affected by recipient 
cytomegalovirus status: 31 of 40 cytomegalovirus negative recipients but only 16 of 36 cytomegalovirus positive recipients survived for 12 months.

Results for related UCB grafts reported from the USA are broadly similar but with less acute GVHD. This may be related to the use of more intensive GVHD prophylaxis. ${ }^{3}$

\section{UNRELATED CORD BLOOD TRANSPLANTATION}

The results for 65 unrelated donor UCB transplantation from the Eurocord registry have also recently been reported. ${ }^{5}$ The patients had a median age of 9 years (range 0.3 to 45 ) and a median weight of $30 \mathrm{~kg}$ (range 4 to 90). Forty one patients had acute or chronic leukaemia. The median follow up was 10 months (range 1 to 30 ). Seven patients had an HLA "identical" donor at HLA-A, -B, and -DRB1; 43 had a one antigen mismatch; 11 had two mismatches; and two had three HLA differences.

Nineteen patients survived for one year. Patients receiving $>3.7 \times 10^{7}$ nucleated cells $/ \mathrm{kg}$ fared better with a survival of $41 \%$ at one year compared with $22 \%$ in those infused with fewer cells. Cytomegalovirus serology was important to outcome: $42 \%$ of cytomegalovirus negative recipients survived 12 months compared with $20 \%$ of cytomegalovirus positive patients. HLA differences did not affect survival. Engraftment was slow and related to the number of nucleated cells $/ \mathrm{kg}$ infused. The probability by day 60 of a neutrophil count $>0.5 \times 10^{9}$ per litre was $87 \%$ and for a platelet count of $>20 \times 10^{9}$ per litre the probability was $39 \%$. Acute GVHD of grade II or more was seen in 21 patients: eight grade II, nine grade III, and four grade IV. The incidence of acute GVHD was significantly less in cytomegalovirus negative recipients. Seven of the 41 patients with leukaemia relapsed.

Results from the USA ${ }^{319} 20$ are better with two year survival of approximately $45 \%$, and more rapid engraftment probably because of the greater use of growth factors posttransplant. There was a significant incidence of acute GVHD. Duke and Minneapolis ${ }^{20}$ reported an incidence of acute GVHD of $\geqslant$ grade II at 35\%; Cairo and Wagner, ${ }^{3}$ in a recent review, reported an incidence of acute GVHD $\geqslant$ grade II of $57 \%$ with $9 \%$ of grade III or IV. In the largest study reported so far involving 272 cases, ${ }^{21}$ Rubinstein et al reported an incidence of acute GVHD of grade III or IV of $23 \%$. The incidence of GVHD did not appear to be related to the apparent degree of HLA mismatch.

\section{COMPARISON WITH UNRELATED BONE MARROW}

TRANSPLANTATION IN CHILDREN

The results of unrelated UCB transplantation compare favourably with results of unmanipulated unrelated BMT. ${ }^{22}$ However, in the UK at least, unmanipulated unrelated donor BMT is rarely if ever performed. The vast majority of British children treated with unmanipulated unrelated BMT receive serotherapy with antithymocyte globulin or monoclonal antibodies, and where appropriate the graft is also lymphodepleted before infusion. In 1996 the Bristol group ${ }^{23}$ reported results for 50 patients with relapsed acute lymphoblastic leukaemia transplanted with manipulated unrelated donor grafts in second complete remission. Event free survival at two years was $53 \% ; 94 \%$ of patients engrafted; acute GVHD $\geqslant$ grade II was seen in $12 \%$ (only three patients had grade III or IV acute GVHD); $26 \%$ relapsed. The same group have more recently reported manipulated unrelated BMT in 15 children and adolescents with poor prognosis Philadelphia positive acute lymphoblastic leukaemia. ${ }^{24}$ All initially engrafted; the incidence of acute GVHD was $13 \%$; two year event free survival was $37 \%$.

\section{Conclusions}

UCB transplantation is feasible. There are apparent limitations owing to the small numbers of progenitor cells present in the harvests. Immunological naivete of cord blood has been demonstrated by the relatively reduced incidence of GVHD in umbilical cord transplants compared with unmanipulated unrelated donor BMT and by the poor outcome in patients who are cytomegalovirus positive before transplantation. However, despite routine prophylaxis, clinically significant acute GVHD was reported in all studies. Follow up is as yet too short to determine whether relapse rates are higher with cord blood than with bone marrow.

Initial results are favourable for matched related UCB transplantation in patients younger than 6 years of age weighing less than $20 \mathrm{~kg}$ who are cytomegalovirus negative. For suitable patients UCB offers the opportunity of prompt haemopoietic stem cell transplantation without any danger or discomfort for the donor. Results in the unrelated setting are not as good as those for children undergoing lymphocyte depleted unrelated donor BMT. However, patients from non-white European populations for whom bone marrow donors are difficult to find on the existing donor panels may benefit from the greater proportion of ethnic minority donors represented in the cord blood banks.

Future results of UCB transplantation will no doubt be better than those summarised above. In vitro expansion of the cord blood progenitor cells may allow heavier patients $(>20 \mathrm{~kg}$ ) to benefit from the immunological naivete of umbilical lymphocytes. ${ }^{25}$ The clinical outcomes need to improve to ensure the future of unrelated cord blood transplantation and to justify the continued investment in cord blood banking. New alternatives are on the horizon. In particular the initial results of mismatched CD34 selected stem cell transplantation are encouraging. ${ }^{26}$

Consultant Paediatric Haematologist, A M WILL

Manchester Children's Hospital,

Pendlbury, Manchester M27 4HA, UK

1 Gluckman E, Broxmeyer H, Auerbach A, et al. Hematopoietic reconstitution in a patient with Fanconi's anaemia by means of umbilical-cord blood from an HLA-identical sibling. N Engl $\mathcal{F}$ Med 1989;321:1174-8.

2 Gluckman E, Rocha V, Boyer-Chammard A, et al. Outcome of cord blood transplantation from related and unrelated donors. $N$ Engl $f \mathrm{Med}$ transplantation

3 Cairo MS, Wagner JE. Placental and/or umbilical cord blood: an alternative source of hematopoietic stem cells for transplantation. Blood 1997;12: 4665-78.

4 Rocha V, Chastang CL, Souillet G, et al. Related cord blood transplants: the Eurocord experience from 78 transplants. Bone Marrow Transplantation 1998;21 (suppl 3):S59-62

5 Gluckman E, Rocha V, Chastang C. European results of unrelated cord blood transplants. Bone Marrow Transplantation 1998;21(suppl 3):S87-91. 6 Stanworth SJ, Will AM. Bone marrow transplantation in children. Current Paediatrics 1998;9:78-82.

7 Broxmeyer HE, Douglas GW, Hangoc G, et al. Human umbilical cord blood as a potential source of transplantable hematopoietic stem/progenitor cells. Proc Natl Acad Sci USA 1989;86:3828-32.

8 Broxmeyer HE, Kurtzberg J, Gluckman E, et al. Umbilical cord blood hematopoietic stem and repopulating cells in human clinical transplantahematopoietic stem and repopulatin

9 Broxmeyer HE, Hangoc G, Cooper S, et al. Growth characteristics and expansion of human umbilical cord blood and estimation of its potential for transplantation in adults. Proc Natl Acad Sci USA 1992;89:4109-13.

10 Rabian-Herzog C, Lesage S, Gluckman E, Charron D. Characterisation of lymphocyte sub populations in cord blood. F Hematother 1993;2:255-7.

11 Bofill M, Akbar AN, Salmon M, et al. Immature CD45-RA ${ }^{\text {low }} \mathrm{RO}^{\mathrm{low}} \mathrm{T}$ cells in the human cord blood. I. Antecedents of CD $45 \mathrm{RA}^{+}$unprimed T cells. $\mathcal{F}$ Immunol 1994;152:5613-23.

12 Risdon G, Gaddy J, Stehman FB, Broxmeyer HE. Proliferative and cytotoxic responses of human umbilical cord blood $\mathrm{T}$ lymphocytes following allogeneic stimulation. Cell Immunol 1994;154:14-24.

13 Cohen SBA, Madrigal JA. Immunological and functional differences between cord and peripheral blood. Bone Marrow Transplantation 1998;21 (suppl 3):S9-12.

14 Indelicato F, Filardi N, Inghuilterra G, et al. Recovery of hemopoietic umbilical cord cells during vaginal and caesarean delivery [abstract]. Bone Marrow Transplantation 1998;21(suppl 3):S122. 
15 Bertolini F, Lazzari L, Lauri E, et al. A comparative study of different procedures for the collection and banking of umbilical cord blood. 7 Hematother dures for the coll
1995;4:29-36.

16 Wagner WE, Broxmeyer HE, Cooper S. Umbilical cord and placental blood hematopoietic stem cells: collection, cryopreservation and storage. $\mathcal{F} \mathrm{Hema}-$ tother 1992;1:167-74.

17 Warwick RM, Barbara JA. Safety aspects of cord blood banking. Bone Marrow Transplantation 1998;21(suppl 3):S40-2.

18 McDonald CP, Robbins S, Hartley S, et al. Cord blood bacteriology: a review of the initial two years. British Association of Tissue Banks. Sixth annual conference, Wakefield, April 1998

19 Kurtzberg JE, Laughlin M, Graham L, et al. Placental blood as a source of hemopoietic stem cells for transplantation into unrelated recipients. $N$ Engl f Med 1996;335:157-66.

20 Wagner JE, Defor T, Rubenstein P, Kurtzberg J. Transplantation of unrelated donor umbilical cord blood: outcomes and analysis of risk factors [abstract]. Blood 1997;90(suppl 1):398A.
21 Rubinstein P, Carrier C, Adamson J, et al. New York Blood Centre's program for unrelated placental/umbilical cord blood (PCB) transprogram for unrelated placental/umbilical cord blood (PCB) transplantation: 243 transp

22 Balduzzi A, Gooley T, Anasetti C, et al. Unrelated donor marrow transplantation in children. Blood 1995;86:3247-56.

23 Oakhill A, Pamphilon DH, Potter MN, et al. Unrelated donor bone marrow transplantation for children with relapsed acute lymphoblastic leukaemia in second complete remission. Br F Haematol 1996;94:574-8.

24 Marks DI, Bird JM, Cornish JM, et al. Unrelated donor bone marrow transplantation for children and adolescents with Philadelphia-positive acute lymphoblastic leukaemia. F Clin Oncol 1998;16:931-6.

25 Kogler G, Callejas J, Sorg RV, Wernet P. An eight fold ex-vivo expansion of long-term culture-initiating cells from umbilical cord blood in stirred suspension cultures. Bone Marrow Transplantation 1998;21(suppl 3):S48-53.

26 Klingebeil $T$, Schumm $M$, Lang $\mathrm{P}$, et al. CD34 enrichment as GVHD prophylaxis in mismatched related and unrelated stem cell transplantation [abstract]. Bone Marrow Transplantation 1998;21(suppl 1):S216.

\section{FETAL AND NEONATAL EDITION}

\section{Fanuary Issue}

The following articles-being published in the January 1999 issue of the Fetal and Neonatal edition of Archives of Disease in Childhood - may be of particular general interest as they relate to community, social, and neurodevelopmental paediatrics.

Clinical outcomes of newborn screening for cystic fibrosis

Donna L Waters, Bridget Wilcken, Les Irwig, Peter Van Asperen, Craig Mellis, fudy Simpson, fohn Brown, Kevin $\mathcal{f}$ Gaskin

Effects of smoking in pregnancy on neonatal lung function

Anthony D Milner, Michael F Marsh, Dorothea M Ingram, Grenville F Fox, Chakraphan Susiva

Systolic blood pressure in babies of less than 32 weeks gestation in the first year of life Win Tin, Northern Neonatal Nursing Initiative

Prevalence and clinical significance of cardiac murmurs in neonates

Sean B Ainsworth, fonathan P Wyllie, Christopher Wren

Examination for cardiac malformations at six weeks of age

Fill Gregory, Alison Emslie, fonathan Wyllie, Christopher Wren

Presentation of congenital heart disease in infancy: implications for routine examination Christopher Wren, Sam Richmond, Liam Donaldson

Antibody response to accelerated Hib immunisation in preterm infants receiving dexamethasone for chronic lung disease

$M \mathcal{F}$ Robinson, F Campbell, P Powell, D Sims, C Thornton

Outcome of mother to infant acquired hepatitis GB-C and G infection

G Zuin, B Saccani, S Di Giacomo, E Tanzi, A R Zanetti, N Principi

"Sucrose analgesia": absorptive mechanism or taste perception?

Luca A Ramenghi, David F Evans, Malcolm I Levene 